\title{
55. Über die Rand- und Eigenwertprobleme der linearen elliptischen Differentialgleichungen zweiter Ordnung.
}

\author{
Von Kunihiko KodaIRA. \\ Physikalisches Institut der Kaiserlichen Universität, Tokyo. \\ (Comm. by T. TAKAGI, M.I.A., May 12, 1944.)
}

$\$ 1$. Vorbereitung ${ }^{1)}$. In vorliegender Note betrachten wir lineare elliptische Differentialausdrücke $L(u)$ für die Funktion $u\left(x^{1}, x^{2}, \ldots, x^{n}\right)$, welche als Eulersche Variationsausdrücke aus einem quadratischen Integral :

$$
E(u)=\int\left(\sum a_{j k} \frac{\partial u}{\partial x^{j}} \frac{\partial u}{\partial x^{k_{c}}}+2 u \sum b_{j} \frac{\partial u}{\partial x^{j}}+c u^{2}\right) d x^{1} d x^{2} \ldots d x^{n}
$$

entstehen, und behandeln die Rand- und Eigenwertprobleme der Differentialausdrücke $L(u)$ für ein beschränktes offenes Gebiet $\mathfrak{M}$ des $\left(x^{1}\right.$, $\left.x^{2}, \ldots, x^{n}\right)$-Raumes nach der Weylschen „Methode der orthogonalen Projektion "2). Dabei sind $a_{j k}, b_{j}, c$ als genügend reguläre, beschränkte Funktionen in $\mathfrak{M}$ vorausgesetzt ${ }^{3)}$, und es soll eine positive Konstante $k$ geben, sodass in jeder Stelle von $\mathfrak{M}$ für beliebige Parameter $u, u_{1}, u_{2}, \ldots, u_{n}$ die Ungleichung :

$$
\sum a_{j k} u_{j} u_{k}+2 u \sum b_{j} u_{j}+c u^{2} \geqq k \sum u_{j}^{2}
$$

gilt. Es ist zweckmässig, $\mathfrak{M}$ als eine Riemannsche Mannigfaltigkeit mit der Metrik: $d s^{2}=g_{j k} d x^{j} d x^{k}$ aufzufassen, welche mit $a_{j k}$ mittels der Relation: $\sqrt{g} g^{j k}=a_{j k}$ verknüpft wird. Dementsprechend setzen wir $b_{j}=\sqrt{\bar{g}} p^{j}, c=\sqrt{\bar{g}} q$; es wird also

$$
E(u)=\int\left(g^{j l} \partial_{j} u \partial_{l o} u+2 u p^{j} \partial_{j} u+q u^{2}\right) \sqrt{g} d G,
$$

wobei $\partial_{j}=\frac{\partial}{\partial x^{j}}$ und $d G=d x^{1} d x^{2} \ldots d x^{n}$ gesetzt wird. Nun führen wir als Hilfsmittel „Skalar-Vektoren“ $\phi, \Psi, \ldots$ mit $n+1$ Komponenten :

$$
\Phi=\left(\varphi, \varphi_{1}, \varphi_{2}, \ldots, \varphi_{n}\right)
$$

ein, und setzen für beliebiges Teilgebiet $G$ aus $\mathfrak{M}$

$$
\begin{gathered}
(u, v)_{G}=\int_{G} u v \sqrt{g} d G, \\
(\varphi, \Psi)_{G}=\int_{G}\left(g^{j k} \varphi_{j} \psi_{k}+\varphi p^{j} \psi_{j}+\phi p^{j} \varphi_{j}+q \varphi \psi\right) \sqrt{g} d G .
\end{gathered}
$$

1) Vgl. R. Courant und D. Hilbert: Meth. d. Math. Physik II, Kap. VII.

2) H. Weyl: Method of orthogonal projections in potential theory, Duke Math. Journ. Vol. 7 (1940), 411-444.

3) Eine Funktion heisst regulär, wenn sie bis zur genügend hohen Ordnung stetig differenzierbar ist. Vgl. J. Hadamard: Lectures on Cauchy's problem, pp. 11-12. 
Für $(u, v)_{\mathfrak{M}}$ bzw. $(\mathscr{\psi}, \Psi)_{\mathfrak{M}}$ schreiben wir $(u, v)$ bzw. $(\Phi, \Psi)$, und setzen $\|u\|=\sqrt{(u, u)},\|\Phi\|=\sqrt{\left.\left(\Phi^{\prime}\right), \Phi\right)}$. Ferner definieren wir den Operator $\mathfrak{a}$ bzw. $a^{*}$ durch

bzw.

$$
\mathfrak{a} u=\left(u, \partial_{1} u, \partial_{2} u, \ldots, \hat{c}_{s} u\right),
$$

$$
\mathfrak{a}^{*} \Phi=-\frac{1}{\sqrt{g}} \partial_{j}\left(\sqrt{g} \varphi^{j}\right)+p^{j}\left(\varphi_{j}-\partial_{j} \varphi\right)+q^{*} \varphi
$$

wobei

$$
q^{*}=q-\frac{1}{\sqrt{g}} \partial_{i}\left(\sqrt{g} p^{j}\right)
$$

ist. In dieser Schreibweise ist also $E(u)=(\mathfrak{a} u, \mathfrak{a} u)$. Der Differentialoperator $L$ lässt sich dann schreiben :

$$
L=\mathfrak{a}^{*} \mathfrak{a}=-\Delta+q^{*}, \quad \Delta=\frac{1}{\sqrt{g}} \partial_{j} \sqrt{g} g^{j k} \partial_{k},
$$

und es gelten die „, Greenschen Formeln “ :

$$
\begin{gathered}
(\Phi, \mathfrak{a} u)_{G}=\left(\mathfrak{a}^{*} \Phi, u\right)_{G}+\int_{\Gamma}\left(\varphi^{j}+p^{j} \varphi\right) u v \sqrt{g} d o_{j}, \\
(v, L(u))_{G}=(L(v), u)_{G}+\int_{\Gamma}\left(v \partial_{j} u-u \partial_{j} v\right) \vee g d o^{j},
\end{gathered}
$$

wobei $I^{\prime}$ die Oberfläche vom Gebiet $G$ ist und $d o_{j}$ die Flächenemente : $(-1)^{j-1} d x^{1} d x^{2} \ldots d x^{j-1} d x^{j+1} \ldots d x^{n}$ bedeutet.

Im Vorhergehenden ist für $u, v, \Phi, \ldots$ natürlich geeignete Regularität vorausgesetzt. Nunmehr lassen wir diese Voraussetzung weg, und betrachten alle nach Lebesgue messbaren Funktionen. Es sei $D(u)$ ein selbst adjungierter Differentialausdruck der Form: $-\Delta u+h u$, wobei $h$ eine in $\mathfrak{M}$ genügend reguläre Funktion sein soll. Dann gilt das

Hauptlemma. Es sei e eine in $\mathfrak{M}$ nach Lebesgue messbare Funktion mit $(e, e)<+\infty$ und es gelte $(e, D(\eta))=0$ für alle in einem. Randstreifen $^{1)}$ von $\mathfrak{M}$ verschwindenden 3 -mal stetig differenzierbaren Funktionen $\eta$. Dann ist $e$ in $\mathfrak{M}$ regulär und genügt der Gleichung $D(e)=0$. Sind ausserdem $g_{j k}$ und h regulär analytisch, so ist auch $e$ regulär analytisch.

Der Beweis dieses Hauptlemmas soll in $\$ 3$ gegeben werden--Nun führen wir die Funktionenräume :

und

$$
\mathfrak{l}=\{u ;\|u\|<+\infty\}
$$

$$
\mathfrak{L}=\{\Phi ;\|\Phi\|<+\infty, \quad\|\varphi\|<+\infty\}
$$

ein. ( $\{* ; E\}$ bezeichnet im allgemeinen die Menge aller * mit der Eigenschaft $E$.) $\mathfrak{l}$ bildet offenbar einen vollständigen Hilbertschen Raum mit der Metrik \|\|$; \mathfrak{Z}$ ist aber in der Metrik \|\| nicht mmer vollständig. Wir setzen

1) Vgl. Courant u. Hilbert: Meth. d. Math. Physik, s. $4 \$ 1$. 


$$
Q(\Phi, \Psi)=(\Phi, \Psi)+(\varphi, \psi) ;
$$

in dieser Metrik $Q$ wird dann $\mathfrak{L}$ auch vollständig. Ferner bezeichnen wir den Funktionenraum aller in einem Randstreifen verschwindenden 3-mal stetig differenzierbaren Funktionen mit $\mathfrak{n}$. Die Räume $\mathfrak{Y}$ und D, die in unserer Theorie zentrale Rolle spielen, definieren wir dann folgendermassen

Definition $I . \quad \mathfrak{V}$ ist die in $\mathfrak{Q}$ gebildete $Q$-abgeschlossene Hülle vom Raum aller $Y=\mathfrak{a} \eta, \eta \in \mathfrak{n}: \mathfrak{Y}=Q$-abg. Hülle von $\{\mathfrak{a} \eta ; \eta \in \mathfrak{n}\}$. $Y \in \mathfrak{Y}$.

Definition II. $\mathfrak{D}$ ist der Raum aller ersten Komponenten $y$ von

Es gilt für jedes $\eta \in \mathfrak{n}$ die bekannte Ungleichung')

$$
\|\eta\| \leqq C\|a \eta\| \text {, }
$$

wobei $C$ eine von $\eta$ unabhängige Konstante ist. Jedes $Y \in \mathfrak{Y}$ genügt also

$$
\|y\| \leqq C\|Y\|,
$$

woraus folgt

$$
\|Y\|^{2} \leqq Q(Y, Y) \leqq\left(1+C^{2}\right)\|Y\|^{2} .
$$

In $\mathfrak{Y}$ ist also die Metrik \|\| mit der Metrik $Q$ äquivalent. $\mathfrak{Y}$ ist also im Sinne der Metrik \|\| auch, ein vollständiger Hilbertscher Raum. Andererseits gilt es wegen (1.3) für beliebiges $\eta \in \mathfrak{n}$ die Gleichung: $\left(\eta, \mathfrak{a}^{*} \Psi\right)=(\mathfrak{a} \eta, \Psi)$. Jedes $Y \in \mathfrak{Y}$ genügt also für beliebiges stetig differenzierbares $\Psi \in \mathfrak{Q}$

$$
\left(y, \mathfrak{a}^{*} \Psi\right)=(Y, \Psi) .
$$

Hieraus folgt, dass jedes $Y \in \mathfrak{Y}$ durch seine erste Komponente $y$ eindeutig bestimmt wird. Man kann also für jedes $Y \in \mathfrak{Y}$

$$
Y=A y
$$

schreiben. Der so definierte Operator $A$ ist offenbar ein abgeschlossener linearer Operator, der den überall dichten Teilbereich $\mathfrak{D}$ aus dem Hilbertschen Raum $\mathfrak{l}$ auf dem Hilbertschen Raum $\mathfrak{Y}$ (mit der Metrik II I) abbildet. $A$ ist nichts anderes als die Abschliessung von a, wenn man $\mathfrak{a}$ als nur in $\mathrm{n}$ definierten Operator betrachtet. Es gilt wegen (1.5), (1.6)

$$
\begin{gathered}
\|y\| \leqq C\|A y\|, \\
\left(y, \mathfrak{a}^{*} \Psi\right)=(A y, \Psi) .
\end{gathered}
$$

Die letzte Gleichung (1.9) zeigt, dass der adjungierte Operator $A^{*}$ von $A$ eine Erweiterung von $a^{*}$ ist.

Definition III. Man setzt $H=A^{*} A$.

Es gilt dann wegen (1.8)

$$
\|y\| \leqq C\|A y\| \leqq C^{2}\|t y\| \text {. }
$$

1) Courant u. Hilbert, S. 484 
$H$ ist offenbar ein positiv-definiter selbst adjungierter Operator mit dem in $\mathfrak{l}$ überall dichten Definitionsbereich, und eine Erweiterung vom in $\mathfrak{n}$ definierten Operator $L=\mathfrak{a}^{*} \mathfrak{a}$. Ferner ist die Abbildung $A y \rightarrow y$ vom $\mathfrak{Y}$ in $\mathfrak{l}$, wie nach Rellich gezeigt wird ${ }^{1}$, vollstetig. Die Abbildung $H y \rightarrow y$ muss also wegen (1.10) um so mehr vollstetig sein. Damit ist bewiesen der folgende:

Satz 1. Der Differentialoperator $L$, der als nur in $\mathfrak{n}$ definierter Operator betrachtet werden soll, besitzt eine positiv-definite selbst adjungierte Erweiterung $H$. Die Umkehrung $H y \rightarrow y$ von $H$ ist vollstetig ${ }^{2}$.

§2. Rand- und Eigenwertprobleme. Das Eigenwertproblem ist schon durch den Satz 1 beinahe gelöst. Es gibt nämlich für $H$ wegen der Vollstetigkeit von $H y \rightarrow y$ ein System der Eigenwerte $\lambda_{j}$ mit $\lambda_{j} \rightarrow+\infty(j \rightarrow \infty)$ und zugehörige Eigenfunktionen $e_{j}$, die zu $\mathfrak{D}$ gèören und einen vollständigen normierten orthogonalen System aus $\mathfrak{l}$ bilden. Für beliebiges $\eta \in \mathfrak{n}$ gilt dabei

$$
\lambda_{j}\left(e_{j}, \eta\right)=\left(H e_{j}, \eta\right)=\left(e_{j} H \eta\right)=\left(e_{j}, L(\eta)\right) .
$$

Setzt man $D=L-\lambda_{j}=-\Delta+q^{*}-\lambda_{j+}$ dann gilt also $\left(e_{j}, D(\eta)\right)=0$. Nach dem Hauptlemma muss also $e_{j}$ in $\mathfrak{M}$ regulär sein, und $L\left(e_{j}\right)-\lambda_{j} e_{j}=$ $D\left(e_{j}\right)=0$ genügen. Damit ist bewiesen der

Satz 2. Es gibt für $L$ ein vollständiges normiertes orthogonales System von Eigenfunktionen $e_{j}$, welche in $\mathfrak{M}$ regulär sind und $z u \mathfrak{D}$ gehören. Die zugehörigen Eigenwerte $\lambda_{j}$ sind alle positiv, und es gilt: $\lim _{j \rightarrow \infty} \lambda_{i j}=+\infty$.

Das Randwertproblem kann nun folgendermassen formuliert werden :

Randwertproblem. Es sei eine in $\mathfrak{M}$ beschränkte, stetig differenzierbare Funktion $f$ mit $\|\mathfrak{a} f\|<+\infty$ gegeben. Gesucht ist dann eine in $\mathfrak{M}$ zweimal stetig differenzierbare Funktion $\varphi$, welche in $\mathfrak{M}$ die Gleichung $L(\varphi)=0$ genügt, und für welche $\varphi-f z u \mathfrak{D}$ gehört.

Wegen der Vollständigkeit von $\mathfrak{Y}$ in Metrik \| \|, kann man af aus $\mathcal{Z}$ in zweien Teilen zerspalten : $\mathfrak{a} f=\Phi+Z$, wobei $Z \in \mathfrak{Y}$ und $(\Phi, Y)=0$ für jedes $Y \in \mathfrak{Y}$ sein soll. Danr ist die erste Komponente $\varphi$ von $\Phi$ gerade die gesuchte Funktion. Denn: Erstens ist es klar, dass $\varphi-f=-z \in \mathfrak{D}$ ist. Zweitens gilt wegen (1.6) für jedes $\eta \in \mathfrak{n}$

$$
(\varphi, L(\eta))=\left(f, \mathfrak{a}^{*} \mathfrak{a} \eta\right)-\left(z, \mathfrak{a}^{*} \mathfrak{a} \eta\right)=(\mathfrak{a} f, \mathfrak{a} \eta)-(Z, \mathfrak{a} \eta)=(\Phi, \mathfrak{a} \eta)=0 .
$$

$\varphi$ ist also nach dem Hauptlemma in $\mathfrak{M}$ regulär und genügt $L(\varphi)=0$. Um die Eindeutigkeit von $\varphi$ zu zeigen, sei $\varphi_{1}$ eine weitere Lösung vom Randwertproblem; man setze $\varphi-\varphi_{1}=\psi$. Dann gilt wegen $\psi \in \mathfrak{D}$ $\mathfrak{a} \psi \in \mathfrak{Y}$, also $(\mathfrak{a} \psi, \mathfrak{a} \eta)=(L(\psi), \eta)=0$ für jedes $\eta \in \mathfrak{n}$. Mithin muss $\psi=0$

1) Courant u. Hilbert, S. 489; vgl. auch Rellich: Cött. Nachr. 1930.

2) K. Friedrichs: Spektraltheorie halbbeschränkter Operatoren, I. Math. Ann. Bd. 109 (1934), S. 486.

3) Courant u. Hilbert, S. 483. 
sein. Es gilt also der

Satz 3. Randwertprobleme besitzen eine und nur eine Lösung. Für analytische Differentialgleichung ist die Lösung auch regulär analytisch.

§3. Beweis des Hauptlemmas. Für den Fall des analytischen Differentialausdrucks der Form $\Delta$, haben wir dieses Hauptlemma in einer vorangehenden Note $^{1)}$ bewiesen. Die dabei benutzte Methode wird durch eine kleine Modifikation auch für allgemeinen nicht analytischen Differentialausdruck anwendbar.

Es sei $e$ ein Element aus $\mathfrak{l}$ und es gelte für alle $\eta \in \mathfrak{n}$ die Gleichung $(e, D(\eta))=0$. Man hat dann zu zeigen, dass $e$ regulär ist und $D(e)=0$ gilt. $D(e)=0$ folgt aber unmittlbar aus $(e, D(\eta))=0$, falls $e$ regulär (mindestens zweimal stetig differenzierbar!) ist. Man hat also nur die Regularität von $e$ zu beweisen-. Es sei $r(x, \xi)$ der geodätische Abstand von $x$ bis $\xi$ im Sinne der Metrik: $d s^{2}=g_{j k} d x^{j} d x^{k}$, und $P(x, \xi)=\{r(x, \xi)\}^{2}$. Man kann dann nach Hadamard ${ }^{2)}$ eine Funktion der Form :

$$
u(x, \xi)=P^{p} U(x, \xi)+(\log P) V(x, \xi), \quad p=-\frac{n-2}{2},
$$

mit regulären $U, V$ mit $U(\xi, \xi)=1$ so konstruieren, dass $D(u)$ in $x$ und $\xi$ auch regulär ist. Wir wählen dann eine genügend kleine positive Konstante $\alpha$, bilden mindestens 4-mal stetig differenzierbaren Funktionen $I I(P), L(P)$ mit

$$
\begin{aligned}
& I I(P)=\left\{\begin{array}{lll}
P^{p} & \text { für } & r \leqq \alpha, \\
0 & \text { für } & r \geqq 2 \alpha,
\end{array}\right. \\
& L(P)=\left\{\begin{array}{lll}
\log P & \text { für } & r \leqq \alpha, \\
0 & \text { für } & r \geqq 2 \alpha,
\end{array}\right.
\end{aligned}
$$

und setzen

$$
\zeta(x, \xi)=\Pi(P) U+L(P) V .
$$

Ferner wählen wir eine positive Konstante $\beta<\alpha$ und setzen

$$
\begin{aligned}
& I I_{\beta}(P)=\left\{\begin{array}{lll}
\frac{1}{2 \beta^{n}}\left\{n \beta^{2}-(n-2) P\right\} & \text { für } & r \leqq \beta, \\
I I(P) & \text { für } & r>\beta,
\end{array},\right. \\
& L_{\beta}(P)=\left\{\begin{array}{lll}
2 \log \beta-1+\frac{P}{\beta^{2}} & \text { für } & r \leqq \beta, \\
L(P) & \text { für } & r>\beta,
\end{array}\right. \\
& \eta_{\beta}(x, \xi)=I I_{\beta}(P) U+L_{\beta}(P) V .
\end{aligned}
$$

$\eta_{\beta}(x)=\eta_{\beta}(x, \xi)$ lässt sich aber durch $\eta \in \mathfrak{n}$ so approximieren, dass

1) K. Kodaira: Über die harmonischen Tenforfelder in Riemannschen Mannigfaltigkeiten, in dieser Proc., §4.

2) J.. Hadam:ard: Lectures on Cauchy's Problem, Book II, Chap. III. 
$\left\|D(\eta)-D\left(\eta_{\beta}\right)\right\| \rightarrow 0$ wird ${ }^{1)}$. Aus $(e, D(\eta))=0$ folgt also $\left(e, D\left(\eta_{\beta}\right)\right)=0$.

Setzt man nun

$$
\delta(\beta ; x, \xi)=D\left(\eta_{\beta}\right)-D(\zeta),
$$

so schreibt sich $\left(e, D\left(\eta_{\beta}\right)\right)=0$ auch in der Gestalt

$$
(e, \delta(\beta))=-(e, D(\zeta)) \text {. }
$$

Für $r(x, \xi)>\beta$ ist aber $\delta(\beta ; x, \xi)=0$ und für $r(x, \xi)<\beta$ gilt

$$
\lim _{\beta \rightarrow 0} \beta^{n} \delta(\beta ; x, \xi)=n(n-2) \quad(\text { gleichmässig in } r(x, \xi)<\beta) \text {. }
$$

Hieraus folgt ${ }^{2)}$

$$
\lim _{\beta \rightarrow 0} \int \mid(e, \delta(\beta ;, \xi))-(n-2) \omega e(\xi) \sqrt{g} d G_{\xi}=0,
$$

wobei $\omega$ den Oberflächeninhalt der $n$-dim. Einheitskugel bedeutet. Es ist also

$$
e(\xi)=-\frac{1}{(n-2) \omega}(e, D(\zeta(, \xi))) .
$$

$D(\zeta)$ ist aber in $x$ und $\xi$ regulär. $e$ muss also auch regulär sein. Im analytischen Fall kann man als $u$ eine Grundlösung von $D(u)=0$ wählen. Für beliebiges Teilgebiet $G$ aus $\mathfrak{M}$ mit dem Rand $I$ gilt dann

$$
e(\xi)=-\frac{1}{(n-2) \omega} \int_{\Gamma}\left\{e \partial_{j} u(, \xi)-u(, \xi) \partial_{j} e\right\} \sqrt{g} d 0^{j},
$$

falls $\xi \in G$ ist. $e$ ist also regulär analytisch. -Damit ist unser Hauptlemma vollständig bewiesen.

Das Hauptlemma lässt sich auf die folgende Form verallgemeinern :

Satz 4. Es sei $D=-\Delta+h$ und $t$ eine in $\mathfrak{M}$ reguläre Funktion. Genügt dann e $\in \mathfrak{l}$ für jedes $\eta \in \mathfrak{n}$ der Gleichung $(e, D(\eta))=(t, \eta)$, so ist auch $e$ in $\mathfrak{M}$ regulär und es gilt $D(e)=t$.

Beweis. Man definiere $\eta_{\beta}$ und $\zeta$ wie oben. So gilt

$$
(e, \delta(\beta))=\left(t, \eta_{\beta}\right)-(e, D(\zeta)),
$$

also folgt wegen (3.1)

$$
(n-2) \omega e(\xi)+(e, D(\zeta(, \xi)))=(t, \zeta(\quad, \xi)) \text {. }
$$

$(t, \zeta)$ lässt sich aber mit regulären Funktionen $F, H$ in der Form

$$
(t, \zeta(\quad, \xi))=\int\{I(P) F(\pi, \xi)+L(P) H(\pi, \xi)\} d \pi^{1} d \pi^{2} \ldots d \pi^{n}
$$

schreiben, wobei $P=g_{j k}(\xi) \pi^{j} \pi^{k}$ ist, wenn man mit $\pi^{j}$ die normalen

1) Vgl. K. Kodaira: a.a.O.

2) Vgl. K. Kodaira: a.a.O. 
Koordinaten um $\xi$ bezeichnet. $(t, \zeta)$ ist also in $\xi$ regulär, $e$ muss also auch regulär sein.

Bemerkung. Mit Hilfe dieses letzten Satzes kann man auch die Randwertaufgabe für die nicht homogene Gleichung $L(\varphi)=t$ lösen : Es sei wieder eine in $\mathfrak{M}$ beschränkte stetig differenzierbare Funktion $f$ mit $\|a f\|<+\infty$ gegeben. Man hat dann zu beweisen, dass es eine in $\mathfrak{M}$ reguläre Funktion $\varphi$ mit $L(\varphi)=t, \varphi-f \in \mathfrak{D}$ gibt. Es gilt wegen (1.8)

$$
|(\mathfrak{a} f, Y)-(t, y)| \leqq(\|\mathfrak{a} f\|+C\|t\|)\|Y\|,
$$

also ist $(\mathfrak{a} f, Y)-(t, y)$ eine beschränkte lineare Funktion von $Y$ aus $\mathfrak{Y}$. Es gibt mithin nach dem Riesschen Satz ein $Z \in \mathfrak{Y}$ mit

$$
(\mathfrak{a} f, Y)-(t, y)=(Z, Y) \text { für alle } Y \in \mathfrak{Y}^{1)} \text {. }
$$

Setzt man nun $\Phi=a f-Z$, so ist $\varphi$ gerade die gesuchte Funktion. Denn: Erstens ist es klar, dass $\varphi-f \in \mathfrak{D}$ ist. Zweitens gilt für jedes $\eta \in \mathfrak{n}$ wegen (3.2)

$$
(\varphi, L(\eta))=\left(f-z, \mathfrak{a}^{*} \mathfrak{a} \eta\right)=(\mathfrak{a} f-Z, \mathfrak{a} \eta)=(t, \eta),
$$

also muss $\varphi$ wegen des obigen Satzes regulär sein und der Gleichung $L(\varphi)=t$ genügen.

1) Dies bedeutet eine Verallgemeinerung der "Methode der orthogonalen Projektion “. Für $t=0$ ist nämlich $Z$ die Projektion von af auf $\mathfrak{V}$. 